11 deletion, corresponding to exons 6 to 37 of ATM, was identified in both siblings. Subsequent Sanger sequencing of $A T M$ identified a second pathogenic variant [c.9091del; p.(Gln3031Lysfs*2)]. The diagnosis was also supported by abnormal chromosome breakage studies.

Clinical laboratories should have clear and consistent reporting policies when determining the significance of deletions involving genes associated with autosomal recessive conditions. This case demonstrates how phenotypic information is critical in guiding what findings should be reported and what follow up testing should be considered if a diagnosis has not been established.

\section{COMPREHENSIVE FISH TESTING USING FFPE TISSUE MICROARRAY TO SCREEN FOR SECONDARY ABNORMALITIES IN MANTLE CELL LYMPHOMA: A RETROSPECTIVE STUDY}

Fiona Webb $^{1}$, Collette Mahler-Hindson ${ }^{2}$, Monica Armstrong ${ }^{1}$, Jacqueline Cosh ${ }^{1}$, RayMun Koo ${ }^{3}$, Nalini Pati ${ }^{3}$,

Adrienne Morey ${ }^{1,2,4}$, Dipti Talaulikar ${ }^{1,2,3}$

${ }^{1}$ Department of Diagnostic Genomics, ACT Pathology,

Canberra, Australia; ${ }^{2}$ Australian National University, Canberra, Australia; ${ }^{3}$ Department of Haematology, ACT Pathology, Canberra, Australia; and ${ }^{4}$ Department of Anatomical Pathology, ACT Pathology, Canberra, Australia

Aim: Mantle cell lymphoma (MCL), is characterised by the $\mathrm{t}(11 ; 14)$. Detection of $\mathrm{t}(11 ; 14)$ by fluorescence in situ hybridisation (FISH) is used in diagnosis. Clinically significance of additional abnormalities remains unclear. Our aim was to use a comprehensive FISH panel to identify secondary changes in a retrospective cohort of MCL samples.

Method: FISH was performed retrospectively on 29 FFPE samples and 4 cell suspensions from bone marrow aspirate diagnosed with MCL. Lymph node punch biopsies were constructed into a tissue microarray of 10 samples per slide in duplicate. The FISH probe panel was designed to detect rearrangements of BCL6, MYC, and BCL2, copy number changes of ATM and TP53 and confirmation of the presence of the $t(11 ; 14)$. Result: 3/33 samples were excluded due to sub-optimal quality, 5 patients had $\mathrm{t}(11 ; 14)$ as the sole abnormality. At least one additional abnormality including losses of TP53 (5/26), ATM $(11 / 26)$, polysomies for MYC (8/26), BCL6 (18/26) and BCL2 (10/26) and rearrangements of MYC (2/26) and BCL6 (1/26) was observed in 26 cases. One patient had triple hit with rearrangements of CCND1, MYC and BCL6.

Conclusion: Expansion of the FISH probe panel detected additional abnormalities in $26 / 30(86 \%)$ of patients including a triple hit case.

\section{RECOMMENDATIONS FROM THE MATERNAL CELL CONTAMINATION WORKING PARTY}

Bruce Bennetts

Department of Molecular Genetics, Children's Hospital at Westmead, Sydney, Australia

Assessment of maternal cell contamination is an important component of prenatal testing and other fetal testing procedures. As well as providing critical information about the purity of fetal samples, MCC assessment also provides an opportunity to verify fetal sample identity. However, a RCPAQAP survey has demonstrated that there are considerable differences between how and when this is done in different laboratories, particularly between cytogenetics laboratories and molecular genetics laboratories.

A workshop was held in Adelaide at the scientific meeting of the Australasian Society of Diagnostic Molecular Genetics (HGSA - Special Interest Group) in April 2019. This workshop brought together representatives from Australian and NZ laboratories and represented both cytogenetics and molecular genetics laboratories. The differences in practise were presented and discussed. It was agreed that any Australasian guidelines should be based around a risk framework. The next step in the process is to get a small group representing cytogenetics and molecular genetics laboratories to draft guidelines before a consultation period involving the RCPA, HGSA and RANZCOG

\section{FISH ANALYSIS OF 17P DELETION: LESSONS LEARNED FROM THE EQA}

\author{
Karen Dun ${ }^{1,2}$ \\ ${ }^{I}$ Victorian Cancer Cytogenetic Service, St Vincent's Hospital, \\ Melbourne, Australia; and ${ }^{2}$ Assessor, Australasian Society of \\ Diagnostic Genomics QAP program, Australia
}

The RCPA/ASDG TP53 quality assurance program (QAP) commenced in 2018 as a pilot program with the implementation of the full program occurring in 2019. The module was devised as a result of the introduction of a new Medicare item allowing laboratories in Australia to bulk bill for TP53 deletion testing in refractory or relapsed chronic lymphocytic leukaemia.

Thirteen laboratories from throughout Australasia participated in the proficiency testing module. The results showed that most laboratories are able to detect TP53 deletions at moderate to low levels with a few exceptions. The data collection that accompanied the QAP program was informative and showed that whilst most laboratories have consistent statistical methods for calculating their laboratory cut-offs for reporting, a clear deficiency was identified in some laboratories regarding the statistical power of their analysis. The RCPA/ASDG TP53 QAP results also showed a definite and long overdue need to revise the NPAAC Cytogenetic guidelines.

\section{HERITABLE CANCER RISK IN THE GENOMIC ERA}

Mandy L. Ballinger, David Thomas

Garvan Institute of Medical Research, Sydney, Australia

Traditional family history based identification of heritable cancer predisposition is giving way to genomic technologies applied to well annotated clinical cohorts, yielding a complex landscape of disease. The International Sarcoma Kindred Study was formed to investigate the heritable aspects of sarcoma. We have previously identified not only monogenic drivers but also polygenic contributions to sarcoma. Most recently we have completed whole genome sequencing (WGS) of 1700 sarcoma probands. A rare variant burden analysis showed the greatest enrichment in pathogenic variation was in TP53, NF1 and POT1. Heritable risk correlates with early age at diagnosis. The Genetic Cancer Risk in the Young Study is a cohort of 1100 young ( $0-40$ years) 